\title{
On the Computation of the Kullback-Leibler Measure for Spectral Distances
}

\author{
Raymond Veldhuis and Esther Klabbers
}

\begin{abstract}
Efficient algorithms for the exact and approximate computation of the symmetrical Kullback-Leibler measure for spectral distances are presented for LPC spectra. A interpretation of this measure is given in terms of the poles of the spectra. The performances of the algorithms in terms of accuracy and computational complexity are assessed for the application of computing concatenation costs in unit-selection-based speech synthesis. With the same complexity and storage requirements, the exact method is superior in terms of accuracy.
\end{abstract}

Index Terms-Concatenation cost, Kullback-Leibler distance, spectral distance measure, speech synthesis, unit selection.

\section{INTRODUCTION}

$\mathbf{O}$ NE PROBLEM of unit-selection-based speech synthesis is the definition of concatenation costs. In [6], these concatenation costs are based on the differences between the spectral envelopes across concatenation boundaries. A symmetrical version of the Kullback-Leibler (SKL) distance, [8], applied to LPC spectra turned out to be the best spectral distance measure for this application. In [7], it is demonstrated that this measure can be successfully applied to define context-sensitive diphones. In [3], [4], experiments are described in which the concatenation errors were subjectively rated on a MOS scale. The results were correlated with a number of spectral distance measures, including the SKL distance. It was found that the SKL distance correlated best with the MOS scores. These findings are confirmed by experiments described in [10].

In unit selection, the concatenation costs are computed at run time. When the concatenation points in the speech database are fixed, such as in [1], calculating the parameters in advance reduces the computational load at run time. This opens the possibility to use parameter sets, such as formant data or the poles of an LPC spectrum, which would otherwise imply too high a computational load. Precalculated parameters, however, require extra storage capacity. This precludes the use of large parameter sets, such as sampled power spectra. The use of a matrix of precalculated distances would eliminate distance calculations at run time completely. However, this would simply require too much storage space, because the speech databases in unit selection can contain as much as one or more hours of speech.

\footnotetext{
Manuscript received April 10, 2001; revised August 28, 2002. The associate editor coordinating the review of this manuscript and approving it for publication was Dr. Hynek Hermansky.

R. Veldhuis is with Twente University, 7500 AE Enschede, The Netherlands (e-mail: R.N.J.Veldhuis@el.utwente.nl).

E. Klabbers is with CSLU, Oregon Graduate Institute of Science and Technology, Beaverton, OR 97006 USA.

Digital Object Identifier 10.1109/TSA.2002.805641
}

In this paper we present methods for efficient exact and approximate computation of the SKL distance between two LPC spectra, given parameters sets of limited size. It is shown that, with the same complexity and storage requirements, the exact method is superior in terms of accuracy. We have chosen LPC spectra, because these are commonly used for modeling the spectral envelopes of speech signals. The exact method can easily be extended to pole-zero spectra.

Another domain, besides speech synthesis, for which the results of this paper are useful, is very-low bit rate speech coding. In this field, the SKL distance can also be used as a distance measure for LPC spectra in the vector quantization of prediction coefficients.

The outline of the paper is as follows. Section II gives a brief introduction to the SKL distance measure for spectral envelopes. Sections III and IV present the exact and the approximate method, respectively. Section V discusses and compares their computational complexity and accuracy. Finally, Section VI gives conclusions.

\section{SymmetricAl KUllbaCK-LEIBLER Distance}

Let $P(\theta)$ and $Q(\theta)$ denote two power-normalized spectral envelopes, i.e.,

$$
\frac{1}{2 \pi} \int_{0}^{2 \pi} P(\theta) \mathrm{d} \theta=\frac{1}{2 \pi} \int_{0}^{2 \pi} Q(\theta) \mathrm{d} \theta=1 .
$$

The Kullback-Leibler distance between $P(\theta)$ and $Q(\theta)$ is defined as

$$
d_{\mathrm{KL}}(P, Q)=\frac{1}{2 \pi} \int_{0}^{2 \pi} P(\theta) \log \frac{P(\theta)}{Q(\theta)} \mathrm{d} \theta
$$

and the symmetrical Kullback-Leibler distance as

$$
\begin{aligned}
d_{\mathrm{SKL}}(P, Q) & =\left(d_{\mathrm{KL}}(P, Q)+d_{\mathrm{KL}}(Q, P)\right) / 2 \\
& =\frac{1}{4 \pi} \int_{0}^{2 \pi}(P(\theta)-Q(\theta)) \log \frac{P(\theta)}{Q(\theta)} \mathrm{d} \theta .
\end{aligned}
$$

The standard procedure for evaluating (3) is by approximating the integral by a sum with $P(\theta)$ and $Q(\theta)$ sampled at equidistant frequencies and subsequently normalized.

\section{EXACT CALCULATION}

Let $r_{P, k}$ denote the normalized autocorrelation function, i.e., with $r_{P, 0}=1$, associated with $P(\theta)$ and $c_{P, k}$ the cepstral coefficients. Then

$$
P(\theta)=\sum_{k=-\infty}^{\infty} r_{P, k} \mathrm{e}^{-\mathrm{j} k \theta}
$$


and

$$
c_{P, k}=\frac{1}{2 \pi} \int_{0}^{2 \pi} \log (P(\theta)) \mathrm{e}^{\mathrm{j} k \theta} \mathrm{d} \theta .
$$

On substitution of (4) and (5) into (3) and by using the symmetry properties of $r_{P, k}$, we obtain

$$
d_{\mathrm{SKL}}(P, Q)=\sum_{k=1}^{\infty}\left(r_{P, k}-r_{Q, k}\right)\left(c_{P, k}-c_{Q, k}\right)
$$

which is the basis of the exact and approximate calculation of $d_{\mathrm{SKL}}(P, Q)$. The derivation is given for LPC (i.e., all-pole) spectra, but can be extended straightforwardly to pole-zero spectra. Let

$$
P(\theta)=\frac{\sigma_{P}^{2}}{\left|A\left(\mathrm{e}^{\mathrm{j} \theta}\right)\right|^{2}} \quad \text { and } \quad Q(\theta)=\frac{\sigma_{Q}^{2}}{\left|B\left(\mathrm{e}^{\mathrm{j} \theta}\right)\right|^{2}}
$$

with $A(z)=1+a_{1} z^{-1}+\cdots+a_{p} z^{-p}$ and $B(z)=1+b_{1} z^{-1}+$ $\cdots+b_{p} z^{-p}$. The $\sigma_{P}^{2}$ and $\sigma_{Q}^{2}$ are chosen such that (1) holds. For notational convenience, we assume $A(z)$ and $B(z)$ to be both of order $p$. The roots of the polynomials $z^{p} A(z)$ and $z^{p} B(z)$ are the poles of the LPC spectrum inside the unit circle of the complex plane. They are denoted by, respectively, $\alpha_{l}$ and $\beta_{l}$, $l=1, \ldots, p$.

We will now derive a closed form for $d_{\mathrm{SKL}}(P, Q)$. Let

$$
\rho_{P, l}=\left(A\left(\alpha_{l}^{-1}\right) \prod_{m \neq l}\left(1-\alpha_{m} \alpha_{l}^{-1}\right)\right)^{-1}, \quad l=1, \ldots, p
$$

denote the residue of $z^{-1} /\left(A(z) A\left(z^{-1}\right)\right)$ at $z=\alpha_{l}$ and let

$$
\rho_{P, l}^{\prime}=\rho_{P, l}\left(\sum_{m=1}^{p} \rho_{P, m}\right)^{-1}, \quad l=1, \ldots, p .
$$

By using (7)-(9) and the Residue Theorem, we may write

$$
r_{P, k}=\frac{1}{2 \pi} \int_{0}^{2 \pi} P(\theta) \mathrm{e}^{\mathrm{j} k \theta} \mathrm{d} \theta=\sum_{l=1}^{p} \rho_{P, l}^{\prime} \alpha_{l}^{k}, \quad k \geq 0
$$

while for the cepstral coefficients we have [9, p. 164]

$$
c_{P, k}=\sum_{l=1}^{p} \frac{\alpha_{l}^{k}}{k}, \quad k \geq 1 .
$$

On substitution of (10) and (11) into (6) and by using $\log (1-$ $x)=\sum_{k=1}^{\infty} x^{k} / k$, we obtain the desired closed form

$d_{\mathrm{SKL}}(P, Q)=\sum_{l=1}^{p}\left(\rho_{P, l}^{\prime} \log \frac{B\left(\alpha_{l}^{-1}\right)}{A\left(\alpha_{l}^{-1}\right)}+\rho_{Q, l}^{\prime} \log \frac{A\left(\beta_{l}^{-1}\right)}{B\left(\beta_{l}^{-1}\right)}\right)$.

This result can be adapted to pole-zero spectra by modifying (8) and (11).

Expression (12) lends itself to an interesting interpretation. Let us assume a source-filter model for the speech, with an all-pole filter modeling the spectral envelope and a noise or pulse source modeling the excitation. The complex poles of the filter model the formants and the other spectral peaks due to the glottal excitation. The real poles model the additional spectral shaping by the glottal excitation. According to (10), $\rho_{P, l}^{\prime}$ expresses the relative importance of the spectral peak associated to $\alpha_{l}$. Therefore, a stronger peak results in a larger contribution of $\rho_{P, l}^{\prime} \log \left(B\left(\alpha_{l}^{-1}\right) / A\left(\alpha_{l}^{-1}\right)\right)$. Because

$$
A\left(\alpha_{l}^{-1}\right)=\overline{A\left(\frac{\mathrm{e}^{\mathrm{j} \arg \left(\alpha_{l}\right)}}{\left|\alpha_{l}\right|}\right)}
$$

in which the horizontal line denotes complex conjugation, $\log \left(B\left(\alpha_{l}^{-1}\right) / A\left(\alpha_{l}^{-1}\right)\right)$ quantifies the spectral mismatch between $1 / B(z)$ and $1 / A(z)$ at the frequency of the pole $\alpha_{l}$, but at a radius $1 /\left|\alpha_{l}\right|$ rather than on the unit circle.

\section{APPROXIMATE CALCULATION}

The approximation of (3) by a sum is straightforward but inefficient. For a more efficient approximation we truncate (6) to a finite sum

$$
d_{\mathrm{SKL}}^{(N)}(P, Q)=\sum_{k=1}^{N}\left(r_{P, k}-r_{Q, k}\right)\left(c_{P, k}-c_{Q, k}\right) .
$$

Because $\left|r_{P, k}\right| \leq p \max _{l}\left(\left|\alpha_{l}\right|\right)^{k}$ and $\left|c_{P, k}\right| \leq p \max _{l}\left(\left|\alpha_{l}\right|\right)^{k}$, cf. (10) and (11), the approximation error of (14) has an upper bound proportional to $\max _{l, m}\left(\left|\alpha_{l}\right|,\left|\beta_{m}\right|\right)^{N}$.

Expression (14) can be computed efficiently by using recursive expressions for $r_{P, k}, r_{Q, k}, c_{P, k}$ and $c_{Q, k}$, based on the prediction coefficients $a_{1}, \ldots, a_{p}$ and $b_{1}, \ldots, b_{p}$. For $k \geq p$, we have the (Yule-Walker) equations

$$
r_{P, k}=-\sum_{l=1}^{p} a_{l} r_{P, k-l}, \quad k \geq p .
$$

When the $r_{P}(1), \ldots, r_{P}(p-1)$ are not available

$$
r_{P, k}=-\sum_{l=1}^{k} a_{l}^{(k)} r_{P, k-l}, \quad k=1, \ldots, p-1
$$

can be used. The $a_{l}^{(k)}, l=1, \ldots, k$, are the prediction coefficients of order $k<p$, which can be computed recursively from the $a_{l}^{(k+1)}$, starting with $a_{l}^{(p)}=a_{l}$. This follows readily from the Durbin algorithm, cf. [5] or a later edition. For the cepstral coefficients we have [9, p. 163]

$$
c_{P, k}=-a_{k}-\frac{1}{k} \sum_{l=1}^{k-1} l c_{P, l} a_{k-l}, \quad k>0
$$

with $a_{k}=0$ for $k>p$.

\section{Computational COMPleXity AND ACCURACY}

The exact method (12) and the approximation $d_{\mathrm{SKL}}^{(N)}(P, Q)$ defined in (14) are compared for the application of computing concatenation costs in unit-selection-based speech synthesis. The approximation of (3) by a finite sum is too inefficient to be a realistic candidate for the approximate calculation of $d_{\text {SKL }}(P, Q)$. The computational complexity depends on the number of parameters available at run time. This is chosen such that the storage requirements of both methods are matching. In addition, the number of terms $N$ in the approximation 
TABLE I

REQUIRED AND ACTUAL PARAMETERS PER CONCATENATION BOUNDARY FOR THE EXACT AND APPROXIMATE METHOD

\begin{tabular}{l|l|l}
\hline \hline & $d_{\mathrm{SKL}}$ & $d_{\mathrm{SKL}}^{(N)}$ \\
\hline Required & $\alpha_{1}, \ldots, \alpha_{p}$ & $a_{1}, \ldots, a_{p}$ \\
\hline Actual & $\alpha_{1}, \ldots, \alpha_{p}$ & $a_{1}, \ldots, a_{p}$ \\
& $a_{1}, \ldots, a_{p}$ & $r_{P, 1}, \ldots, r_{P,\left\lfloor\frac{3}{2} p\right\rfloor}$ \\
& $\rho_{P, 1}^{\prime}, \ldots, \rho_{P, p}^{\prime}$ & $c_{P, 1}, \ldots, c_{P,\left\lfloor\frac{3}{2} p\right\rfloor}$ \\
& $A\left(\alpha_{1}^{-1}\right), \ldots, A\left(\alpha_{p}^{-1}\right)$ & \\
\hline \hline
\end{tabular}

$d_{\text {SKL }}^{(N)}(P, Q)$ is chosen such that the numbers of floating-point operations of the approximate and the exact method are also matching. The choices on the parameters made for both methods are described below.

For the exact computation of (12), the minimum requirement is the availability at run time of the $p$ poles. In addition, the prediction coefficients are provided, which improves the efficiency by $O\left(p^{2}\right)$ operations. A substantial further improvement is obtained by providing the normalized residues (9) and the $A\left(\alpha_{l}^{-1}\right)$ and $B\left(\beta_{l}^{-1}\right)$, leaving only the "cross functions" $A\left(\beta_{l}^{-1}\right)$ and $B\left(\alpha_{l}^{-1}\right)$ to be evaluated at run time. In total, $4 p$ real numbers per concatenation boundary are computed off-line and stored. The complex $\alpha_{j}$ and $\beta_{j}$ occur in conjugated pairs. Therefore, the terms of (12) also occur in conjugated pairs. This has been taken advantage of in the implementation. The final number of floating-point operations required for the exact method depends slightly on the number of real poles. Its computational complexity increases quadratically with $p$.

For the computation of (14), the minimum requirement is the availability at run time of the $p$ prediction coefficients. The computational complexity is further reduced by storing the first $\lfloor(3 / 2) p\rfloor$ normalized autocorrelation coefficients and the first $\lfloor(3 / 2) p\rfloor$ cepstral coefficients. In this way, the storage requirements of the approximate method match those of the exact method and the computation of the first $\lfloor(3 / 2) p\rfloor$ terms only cost 4 floating-point operations per term. The computational complexity of the approximate method increases linearly with $p$. The required parameters and the actual parameters as chosen for the comparison are summarized in Table I.

The accuracy and computational complexity of the exact and the approximate method have been assessed for LPC spectral envelopes estimated from recorded vowels /a/ and /i/. The sampling frequency was $8 \mathrm{kHz}$. The order of prediction $p$ was 10 in all cases. The vowel /a/ was uttered by a male and the vowel /i/ by a female speaker. One LPC spectral envelope, A, was estimated from the vowel /a/. Two very similar LPC spectral envelopes, I1 and I2, were estimated from nonoverlapping subsequences of the vowel /i/.

The SKL distances between the envelopes I1 and I2 and between A and I 2 were computed. This gives an impression of the performance for similar and distinct spectral envelopes. Calculation by the exact method required 971 floating-point operations for both cases. The number of terms $N$ in (14) was set
TABLE II

RESULTS FOR THE EXACT SKL DisTANCE AND THE APPROXIMATION $d_{\mathrm{SKL}}^{(N)}$ WITH $N=24$

\begin{tabular}{l|l|l|l|l}
\hline \hline Envelopes & $d_{\text {SKL }}$ & $d_{\text {SKL }}^{(24)}$ & abs. err. & rel. err. \\
\hline I1,I2 & 0.0042 & 0.0029 & 0.0013 & 0.31 \\
I1,A & 3.7305 & 3.1614 & 0.5691 & 0.15 \\
\hline \hline
\end{tabular}

equal to 24, in which case the number of floating-point operations was as close as possible to that of the exact method. The results and the absolute and relative errors are shown in Table II.

In [7], the authors studied the capability of the SKL distance to detect audible concatenation errors. It can be concluded from their data that a relative error of $15 \%$ or $30 \%$ as in Table II would seriously hamper the detection. This renders the finite sum approximation (14) unsuitable as a low-complexity alternative for the exact method (3) for the application of computing concatenation costs in unit-selection-based speech synthesis.

The number of 971 floating-point operations required for the exact method is still high compared with, for instance, the Euclidian distance between Mel-frequency cepstral coefficients (MFCCs) [2], with the MFCC's available at run time. In that case, the number of floating-point operations is of the order of the number of MFCCs and usually less than 20, but the prediction of audible concatenation errors will be poorer, [3], [4], [6].

Note that the exact method requires that the poles of the LPC spectra be available at run time. The computation of these poles is computationally demanding. Therefore, when in some application these poles are not available, the comparison may have a different outcome.

\section{CONCLUSION}

One exact and one approximate method of computing the symmetrical Kullback-Leibler distance for all-pole spectra were presented. These methods can be used for the computation of concatenation costs in unit-selection based speech synthesis. The exact method is given in a closed form. It requires the availability at run time of the poles of the spectrum, but its performance can be improved by providing a four times larger set of parameters. The approximate method is a truncated infinite sum. It requires the availability of the prediction coefficients and, preferably, a number of autocorrelation and cepstral coefficients. The computational complexity of both methods and the accuracy of the approximate method were assessed for the application of computing concatenation costs in unit-selection-based speech synthesis. The computational complexity of the exact method is substantially higher than that of the Euclidian distance between MFCCs, which is an alternative method. However, the SKL distance is better at predicting audible concatenation errors and the higher complexity seems to be the price one has to pay. When the computational complexity and storage requirements of the approximate method are set to match those of the exact method, its accuracy is too low for this method to be a useful alternative in unit selection. Therefore, the exact method appears to be the optimal choice. 


\section{REFERENCES}

[1] M. Balestri, A. Pacchiotti, S. Quazza, P. Salza, and S. Sandri, "Choose the best to modify the least: A new generation of concatenative synthesis systems," in Proc. Eurospeech 1999, Budapest, Hungary, 1999, pp. 2291-2294.

[2] A. Conkie and S. Isard, "Optimal coupling of diphones," in Progress in Speech Synthesis, J. van Santen, R. Sproat, J. Olive, and J. Hirschberg, Eds. New York: Springer-Verlag, 1997, pp. 293-304.

[3] M. C. Founda, A. I. Chalamandaris, G. D. Tambouratzis, and G. V. Carayannis, "Studying the factors affecting the optimal unit selection algorithm for a TTS system for the Greek language," in Proc. 4th Eur. Conf. Noise Control EURONOISE 2001, Patras, Greece, 2001.

[4] M. Founda, G. Tambouratzis, A. Chalamandaris, and G. Carayannis, "Reducing spectral mismatches in concatenative speech synthesis via systematic database enrichment," in Proc. 7th Eur. Conf. Speech Communication and Technology EUROSPEECH 2001, vol. 2, Aalborg, Denmark, 2001, pp. 837-840.

[5] G. H. Golub and C. F. van Loan, Matrix Computations. London, U.K.: North Oxford Academic, 1986.

[6] E. Klabbers and R. Veldhuis, "On the reduction of concatenation artefacts in diphone synthesis," in Proc. 5th Int. Conf. Spoken Language Processing (ICSLP'98), vol. 5, Sydney, Australia, 1998, pp. 1983-1986.

[7] _ _ "Reducing audible spectral discontinuities," IEEE Trans. Speech Audio Processing, vol. 9, pp. 39-51, Jan. 2001.

[8] S. Kullback and R. Leibler, "On information and sufficiency," Ann. Math. Statist., vol. 22, pp. 79-86, 1951.

[9] L. Rabiner and B. H. Juang, Fundamentals of Speech Recognition Englewood Cliffs, NJ, 1993.

[10] Y. Stylianou and A. K. Syrdal, "Perceptual and objective detection of discontinuities in concatenative speech synthesis," in Proc. IEEE ICASSP'01, vol. 2, Salt Lake City, UT, 2001, pp. 837-840.

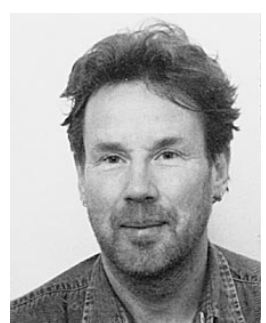

Raymond Veldhuis studied electrical engineering at Twente University, Enschede, The Netherlands. He received the Engineer degree in 1981. In 1988, he received the Ph.D. degree from Nijmegen University on a dissertation entitled "Adaptive Restoration of Lost Samples in Discrete-Time Signals and Digital Images.'

From 1982 to 1998 he worked as a Researcher at Philips Research Laboratories, Eindhoven, The Netherlands, in various areas of digital signal processing, such as audio signal restoration and audio source coding. From 1992 to 2001 he worked at the IPO (Institute of Perception Research), Eindhoven. He is now an Associate Professor at Twente University. He is author of various papers and patents in the field of sound, image and speech processing. He is co-author of the books An Introduction to Source Coding (Englewood Cliffs, NJ: Prentice Hall) and Restoration of Lost Samples in Digital Signals (Englewood Cliffs, NJ: Prentice-Hall). His expertise involves digital signal processing for audio and audio source coding and speech, in particular speech synthesis. He has been active in the development of MPEG standards for audio source coding.

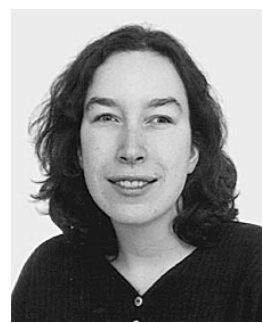

Esther Klabbers studied language and computer science at the Department of Language and Speech, University of Nijmegen, The Netherlands. She received the Masters degree in 1995. In 1996 she joined IPO, at that time known as the Institute for Perception Research, as a Ph.D. student. In 2000, she received the Ph.D. degree from the Eindhoven University of Technology on a dissertation entitled "Segmental and Prosodic Improvements to Speech Generation." From 2000 and 2001, she was a Postdoctoral Researcher at IPO. Currently, she is a Postdoctoral Researcher at the OGI School of Science and Engineering at OHSU in Portland, OR.

Her expertise involves many aspects of speech synthesis, such as prosodic modeling, corpus design, quality evaluation, and applications. 